\title{
Prospective Study of Moxifloxacin prophylaxis in Patients Suffering with HIV Having Contact History of Multidrug Resistant Tuberculosis (MDR-TB)
}

\section{Kalyan Kumar PV ${ }^{1 *}$, Ramakrishna Gorantla ${ }^{2}$ and Ramakrishna Rachakonda}

${ }^{1}$ Department of Pulmonary Medicine, Katuri Medical College, Guntur, India

${ }^{2}$ Department of General Medicine, Katuri Medical College, Guntur, India

\begin{abstract}
Aim and Objectives: To find out the effectiveness of moxifloxacin prophylaxis in HIV patients having contact history of MDR-TB.

Materials and Methods: This is an observational prospective cohort study of HIV patients divided in to two groups, moxifloxacin prophylaxis group and non-prophylaxis group. These groups are followed for four years from March 2012 to March 2016 to appraise the incidence of tuberculosis and mortality in these two cohorts.

Results: The tuberculosis incidence in more in non-prophylaxis group when compared to prophylaxis group. Crude tuberculosis incidence is 275 per 1000 person/years in non-prophylaxis group when compared to 42 per 1000 person/ years in prophylaxis group $(p<0.005)$. Incidence of MDR-TB is more in non-prophylaxis group (26 vs $6 ; p=0.002)$ when compared to prophylaxis group. Crude mortality rate is more in non-prophylaxis group (774 vs 103; $p=0.003$ ) when compared to the other group which was further confirmed by Cox regression hazards model. Proportional hazards model in which moxifloxacin prophylaxis had a $60 \%$ decrease in mortality compared to non-prophylaxis (adjusted relative hazard).
\end{abstract}

Conclusion: Prophylaxis with 6-months moxifloxacin is an effective alternative for HIV patients instead of isoniazid suspected to contact with multidrug resistant tuberculosis.

Keywords: Multi drug resistant tuberculosis; Tuberculosis; Human immunodeficiency virus; Extensive drug resistant tuberculosis; Fluoroquinolone; Acquired immunodeficiency syndrome; Antiretroviral therapy

\section{Introduction}

HIV/AIDS is a global pandemic. As of March 2016, 36.7 million (34.0 to 39.8 million) people globally were living with HIV. Nearly 2.1 million (1.8 to 2.4 million) people became newly infected with HIV. Despite the impressive roll-out of antiretroviral therapy (ART) programmes worldwide including in low and middle-income countries, 1.1 million (940,000 to 1.3 million) people died from AIDS related illnesses [1].

Tuberculosis (TB) is a leading killer of HIV-positive people: one in three HIV deaths was due to TB. At least one-third of people living with HIV worldwide were infected with TB bacteria. People living with HIV are 20 to 30 times more likely to develop active TB disease than people without HIV. HIV and TB form a lethal combination, each speeding the other's progress. About 0.4 million people died of HIV associated TB. Approximately one third of deaths among HIV-positive people were due to TB [2].

According to World Health Organization (WHO), nearly 50\% of the world's burden of multi-drug resistant tuberculosis (MDR-TB) cases are present in India. The situation of TB is further threatened by the overwhelming effect of human immunodeficiency virus (HIV) on tuberculosis. Tuberculosis is one of the earliest opportunistic diseases to develop amongst persons infected with HIV and HIV infection is the most powerful risk factor of progression of TB [3]. The $4^{\text {th }}$ World Health Organization report on anti-tuberculosis drug resistance, MDR-TB has been shown to be almost twice as common in TB patients living with HIV compared to TB patients without HIV [3].

The intersection of the HIV and TB epidemics resulted in a dramatic upsurge in global TB incidence. In high income countries, all forms of TB are regarded as AIDS-defining conditions [4]. In contrast, the WHO staging system, separates pulmonary TB (stage 3 ) from extra pulmonary TB (stage 4 or AIDS) [5]. Since TB commonly occurs in non immunocompetent people, it is not surprising that $\mathrm{TB}$ occurs with a wide range of $\mathrm{CD} 4$ counts. The $\mathrm{CD} 4$ count determines the incidence as well as the clinical and radiographic presentations of TB.

HIV-infected patients are at greatly increased risk of developing active TB from reactivated latent tuberculosis infection (LTBI). HIV infection is also a risk factor for accelerated progression of TB following exposure, which has resulted in outbreaks of multidrug-resistant (MDR) and extensively drug-resistant (XDR-TB) [5].

Likewise, TB seems to have a negative impact on HIV disease, increasing the risk of progression to AIDS or death following TB treatment. The acceleration of HIV diseases by TB may result from one or more of the following mechanisms:

1. TB infection is associated with significant increases in plasma HIV viremia. As is the case with other opportunistic infections. Persistently high levels of viremia have been observed in patients despite initiation of effective antitubercular therapy [6].

2. Generalized immune activation, due to TB infection, may increase the proportion of $\mathrm{CD} 4$ cells that are preferential targets for HIV.

*Corresponding author: Kalyan Kumar PV, Department of Pulmonary Medicine, Katuri Medical College and Hospital, Guntur, India, Tel: +91-7093261025; E-mail: drpvkalyan@hotmail.com

Received June 22, 2016; Accepted October 28, 2016; Published October 31 2016

Citation: Kalyan Kumar PV, Ramakrishna G, Ramakrishna R (2016) Prospective Study of Moxifloxacin prophylaxis in Patients Suffering with HIV Having Contact History of Multidrug Resistant Tuberculosis (MDR-TB). J Pulm Respir Med 6: 377. doi: 10.4172/2161-105X.1000377

Copyright: () 2016 Kalyan Kumar PV, et al. This is an open-access article distributed under the terms of the Creative Commons Attribution License, which permits unrestricted use, distribution, and reproduction in any medium, provided the original author and source are credited. 
Citation: Kalyan Kumar PV, Ramakrishna G, Ramakrishna R (2016) Prospective Study of Moxifloxacin prophylaxis in Patients Suffering with HIV Having Contact History of Multidrug Resistant Tuberculosis (MDR-TB). J Pulm Respir Med 6: 377. doi: 10.4172/2161-105X.1000377

Page 2 of 6

3. Increased expression of the HIV co-receptors CCR5 and CXCR4 occurs in HIV-infected patients with TB co infection.

In the context of HIV infection, tuberculin skin test (TST) positivity is defined by induration of $\geq 5 \mathrm{~mm}$. The utility of a TST depends on the immune status of the patient and the prevalence of TB in the community. Tuberculin skin testing is not of value for diagnosing active TB in adults in areas where TB prevalence is high because the prevalence of positive skin tests is high in the general population. In low TB prevalence settings, a positive tuberculin skin test in a patient with compatible symptoms and signs of TB may support the diagnosis of TB. A positive tuberculin skin test is an indication for preventive therapy in people not yet on antiretroviral therapy once active TB has been excluded. Treatment of latent TB is associated with two important benefits:

1. Reduction in the likelihood of progression to active TB disease and

\section{Reduction in TB transmission}

Treatment of LTBI reduces the risk of progression to active TB disease, reducing TB-related morbidity and mortality. Among patients in Asia with HIV and LTBI, the risk of developing active TB disease is 5 to 10 percent per year. Overall, treatment of LTBI in individuals with HIV infection reduces active TB disease by 62 percent and mortality by 26 percent [7]. The benefit is greatest among those with positive test for LTBI [7]. Treatment of LTBI also reduces TB transmission. Treatment of LTBI in HIV-infected persons has been strongly recommended for many years by expert panels. However, despite consistent and clear recommendations from authoritative agencies, including the World Health Organization, it is estimated that only 1.3 percent of all HIVinfected patients with LTBI worldwide receive treatment.

According to CDC guidelines, LTBI treatment is warranted for HIV-infected patients in the following circumstances [8]:

1. Individuals with recent contact with a person with active tuberculosis $(\mathrm{TB})$ disease

2. Individuals with a history of inadequately treated healed TB (e.g., fibrotic disease on chest radiograph), regardless of test results for LTBI

3. Individuals with evidence of LTBI by tuberculin skin test

4. Individuals living in resource-limited areas of high TB incidence where testing for LTBI is not available

Isoniazid monotherapy is the preferred agent for treatment of LTBI because of its favorable toxicity profile and treatment completion rates. The optimal duration of therapy is uncertain. Duration of nine months has been recommended for HIV-infected patients in resource-rich settings. Some experts consider six months of isoniazid to be an acceptable alternative. Isoniazid should be supplemented with pyridoxine at a dose of $25 \mathrm{mg} /$ day to prevent peripheral neuropathy. Isoniazid plus rifapentine (given as directly observed doses together once weekly for 12 weeks) may be a reasonable alternative regimen for otherwise healthy HIV-infected patients with LTBI who are not on ART. This is an attractive option for patients who are unable to adhere to nine months of daily therapy with isoniazid. However, HIV-infected patients on ART should not be treated with isoniazid plus rifapentine since there are few data on drug-drug interactions. Rifampin plus pyrazinamide should not be used for treatment of LTBI due to hepatotoxicity.

In HIV, individuals known or assumed to be infected with singledrug INH or rifampicin-resistant organisms, the drug to which the source-case organism is susceptible should be administered as a single agent. For patients exposed to (and presumed to be infected with) MDR$\mathrm{TB}$, treatment should be individualized based on the susceptibility profile of the index case. There are limited data on the effectiveness of treatment of LTBI in persons presumed or known to be infected with MDR-TB. In general, one or two drugs to which the organism from the source case is sensitive should be chosen example, a fluoroquinolone alone (such as levofloxacin) or a combination of a fluoroquinolone and ethambutol.

Pyrazinamide (PZA) given with a second drug has been associated with individual cases of severe hepatotoxicity and should be avoided. A fluoroquinolone-based treatment regimen may be effective in preventing tuberculosis in MDR-infected contacts with fluoroquinolone susceptibility. Duration of treatment has not been studied but 6 to 12 months is reasonable [9].

Guntur district, which forms part of the AP state capital region, tops the list of HIV positive cases in the state. The district also had the dubious distinction of being the "HIV capital" in undivided Andhra Pradesh in the last two years [10]. The following study took place in tertiary health center in Guntur, Andhra Pradesh, India. The main objective of the study was to test the efficacy of the moxifloxacin based prophylaxis for the HIV patients with contact history of MDR-TB or suspected MDR-TB patients.

\section{Materials and Methods}

\section{Recruitment of human samples}

Participants are both non-hospitalized and hospitalized HIVinfected patients who are:

1. Residents of a building on the floor on which a MDR-TB casepatient resided or visited during the infectious period

2. Members of the immediate family, close social contacts, or others (e.g., hospital roommates, some health-care providers) who shared the same indoor environment with an infectious MDRTB patient for substantial periods

A total of 244 study participants who are HIV (HIV-1/HIV-2) infected, ART naïve with $\mathrm{CD} 4$ count $>500$ cells/ $\mu \mathrm{L}$ with MDR-TB contact history are included in the study (Figure 1). LTBI among these contacts was diagnosed using the Mantoux tuberculin skin test using $\geq$ $5 \mathrm{~mm}$ as cut-off. Among $244 \mathrm{HIV}$ patients who are exposed to MDRTB case, 114 subjects have negative tuberculin skin test and 11 subjects with history of active tuberculosis prior to enrolment in the study trial were excluded. The following inclusion criteria was used:

1. HIV patients with contact history of MDR-TB case.

2. Not started on ART.

3. CD4 count $>500$ cells $/ \mu \mathrm{L}$.

4. Mantoux tuberculin skin test $\geq 5 \mathrm{~mm}$.

5. Aged more than 20 years, and recruited from voluntary counseling and testing (VCTC).

6. No evidence of active TB or MDR-TB.

7. Absence of any serious underlying disease.

All contacts are carefully evaluated using symptom review, clinical examination, and chest X-ray (CXR) before initiating treatment. Study participants provided written informed consent for data collection. Demographic information, history of TB exposure, clinical data, co 
Citation: Kalyan Kumar PV, Ramakrishna G, Ramakrishna R (2016) Prospective Study of Moxifloxacin prophylaxis in Patients Suffering with HIV Having Contact History of Multidrug Resistant Tuberculosis (MDR-TB). J Pulm Respir Med 6: 377. doi: 10.4172/2161-105X.1000377

Page 3 of 6

morbidities and CXR findings were abstracted onto data collection forms. For contacts aged $\geq 20$ years, baseline co-morbidities were assessed by measuring random glucose to detect diabetes mellitus (DM), serum hepatic aminotransferase levels and other liver function tests and renal functional tests are obtained.

Thus 117 patients met the criteria for inclusion in our study. Sixty four patients completed the study with moxifloxacin or ethambutol or ethionamide based prophylaxis for 6 months (Figure 1). The remaining 53 patients did not take the above mentioned prophylaxis and continued to take 6 months isoniazid (INH) prophylaxis. Treatment for MDR-LTBI was given by daily directly observed therapy (DOT) for 6 months. The reasons for not taking the fluoroquinolone-based therapy in non-prophylaxis group are:

1. Lack of safety information for Moxifloxacin-based prophylaxis.

2. Preventive therapy with Isoniazid, which is cost-effective when compare to Fluoroquinolone based prophylaxis.

3. Refused prophylaxis.

The patients are offered 6 months fluoroquinolone-based prophylaxis when compared to 1 year prophylaxis due to:

1. The lengthy duration of treatment reduces patient compliance, while the potential occurrence of serious adverse events, further discourages patients and providers acceptance of this therapy.

2. There are no randomized control trials regarding the duration of therapy.

3. As a result, there has been considerable interest and many evaluations of alternative shorter regimens.

The study started enrolling subjects in March 2012 and was completed in March 2016 in a tertiary health centers in Andhra Pradesh.

Ethical approval was obtained from the Institutional Review Board.

\section{Design of the study}

This is a prospective observational cohort study following HIV patients with MDR-TB contacts from March 2012 to March 2016. We performed descriptive analyses to characterize the population. Kaplan-Meier curves are plotted for univariate analysis of tuberculosis incidence separately and mortality in HIV patients separately. Cox proportional hazard model was used to estimate hazard ratio (HR) for recurrent mortality and active tuberculosis between two groups. Both

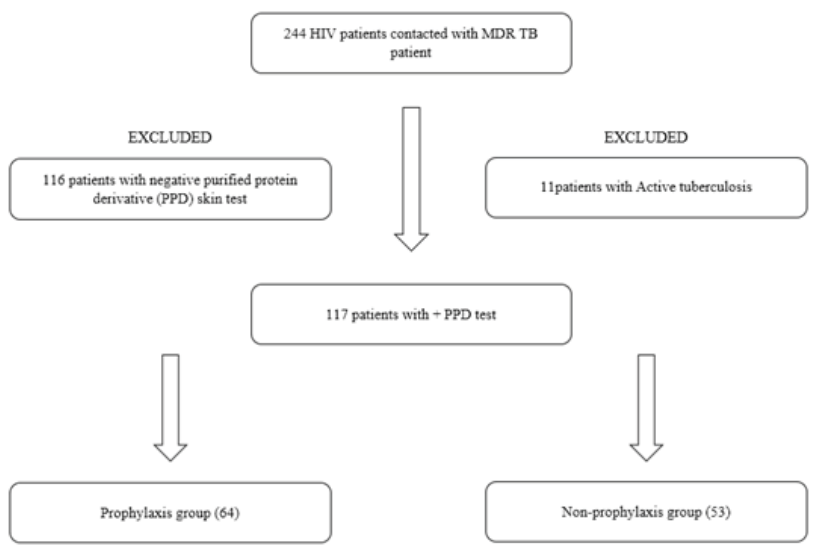

Figure 1: Recruitment of human samples. crude and adjusted analyses from the Cox model are reported. Data were analyzed using SPSS version 24 (IBM SPSS Statistics).

\section{Results}

A total of 244 study participants who are HIV infected, ART naive with CD 4 count $>500$ cells $/ \mu \mathrm{L}$ are studied prospectively for 4 years. Among 244 HIV patients who are exposed to MDR-TB case, 114 subjects have negative purified protein derivative (PPD) skin test and 11 subjects with history of active tuberculosis prior to enrolment in the trial are excluded (Figure 1).

Thus, 117 patients met the criteria for inclusion in our study. Sixty four patients completed the 6 months MDR-TB prophylaxis regimen. The remaining 53 patients did not take the fluoroquinolone (FQ)-based regimens prophylaxis. These 53 patients who refused to take prophylaxis are offered the INH based prophylaxis for 6 months. No subject died during the 6 months of study. The group started on the FQ-based regimens prophylaxis for 6 months are assembled under prophylaxis group. Other group not taken prophylaxis even after exposure to MDRTB case are congregated under non-prophylaxis group.

\section{Baseline characteristics of the patients}

There were more males $70(60 \%)$ than females $47(40 \%)$, and more subjects $81(71 \%)$ with CD4 count between $500-1000$ cells/ $\mu \mathrm{L}$ than those with CD4 count $>1000$ cells/ $\mu \mathrm{L}$ (36.29\%). The median baseline CD4 count was 850 cells/ $\mu \mathrm{L}$ in the beginning of our study. Thirty five (30\%) patients have BMI $>18.5 \mathrm{~kg} / \mathrm{m}^{2}$. Whereas, the majority of patients (82; 70\%) have BMI $<18.5 \mathrm{~kg} / \mathrm{m}^{2}$. The patients are grouped under four classes namely 20-29, 30-39, 40-49 and more than 50 years. Majority of the patients $(34 ; 29 \%)$ are in the age of $30-39$ years (Table 1$)$.

\section{Incidence of tuberculosis}

Nine patients developed active tuberculosis during 213 person/ years of follow up in prophylaxis group and 35 patients developed active tuberculosis during 127 person/years of follow up in non-prophylaxis group. The crude TB incidence per 1000 person/years in prophylaxis group was 42 when compared to 275 in non-prophylaxis group. Among the different age groups, 19 patients with age greater than 50 years developed active tuberculosis during 57 person/years of follow up with crude incidence rate of 331 per 1000 person/years. The incidence of tuberculosis is 4 during 118 person/years of follow up in 20-29 years age group. The crude incidence per 1000 person/years is 34 in this age group (Table 2).

As elderly patients in developing countries suffer from poverty, malnutrition, tobacco smoking, and decreased immunity which are proven risk factors for tuberculosis. Incidence of MDR-TB is more in non-prophylaxis group is more when compared to the prophylaxis

\begin{tabular}{|c|c|c|c|c|}
\hline \multicolumn{2}{|c|}{ Characteristics } & $\begin{array}{c}\text { No of patients } \\
\text { (117) }\end{array}$ & $\begin{array}{l}\text { Prophylaxis } \\
\text { group (64) }\end{array}$ & $\begin{array}{l}\text { Non-prophylaxis } \\
\text { group (53) }\end{array}$ \\
\hline \multirow{4}{*}{ Age in years } & $20-29$ & 33 & 16 & 17 \\
\hline & $30-39$ & 26 & 14 & 12 \\
\hline & $40-49$ & 34 & 23 & 11 \\
\hline & $>50$ & 24 & 11 & 13 \\
\hline \multirow{2}{*}{ Gender } & Male & 70 & 37 & 33 \\
\hline & Female & 47 & 27 & 20 \\
\hline \multirow{2}{*}{$\begin{array}{l}\text { Baseline CD4+ cell } \\
\text { count in cells/ } \mu \mathrm{L}\end{array}$} & $500-1000$ & 81 & 41 & 40 \\
\hline & $>1000$ & 36 & 23 & 13 \\
\hline \multirow{2}{*}{$\begin{array}{l}\text { Body mass index, } \\
\qquad \mathrm{kg} / \mathrm{m}^{2}\end{array}$} & $<18.5$ & 82 & 42 & 40 \\
\hline & $>18.5$ & 35 & 22 & 13 \\
\hline
\end{tabular}

Table 1: Baseline characteristics of the patients. 
Citation: Kalyan Kumar PV, Ramakrishna G, Ramakrishna R (2016) Prospective Study of Moxifloxacin prophylaxis in Patients Suffering with HIV Having Contact History of Multidrug Resistant Tuberculosis (MDR-TB). J Pulm Respir Med 6: 377. doi: 10.4172/2161-105X.1000377

Page 4 of 6

\begin{tabular}{|c|c|c|c|c|c|c|}
\hline \multicolumn{2}{|c|}{ Age } & \multirow{2}{*}{$\begin{array}{c}\text { Total number } \\
64\end{array}$} & \multirow{2}{*}{$\begin{array}{c}\text { Person years } \\
213.38\end{array}$} & \multirow{2}{*}{$\begin{array}{c}\text { TB/MTB incidence } \\
9\end{array}$} & \multirow{2}{*}{$\begin{array}{c}\text { Incidence proportion } \\
34.37\end{array}$} & \multirow{2}{*}{$\begin{array}{c}\text { TB incidence (per } 1000 \\
\text { person-years) }\end{array}$} \\
\hline & Completed & & & & & \\
\hline 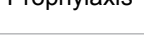 & Not Completed & 53 & 127.44 & 35 & 77.35 & 274.63 \\
\hline \multirow{4}{*}{ Age } & $20-29$ & 33 & 118.34 & 4 & 12.12 & 33.8 \\
\hline & $30-39$ & 26 & 97.23 & 6 & 23.07 & 61.7 \\
\hline & $40-49$ & 34 & 67.9 & 14 & 41.17 & 206.18 \\
\hline & $>50$ & 24 & 57.35 & 19 & 79.16 & 331.29 \\
\hline \multirow{2}{*}{ Gender } & Male & $70(60 \%)$ & 192.36 & 31 & 26.49 & 161.15 \\
\hline & Female & $47(40 \%)$ & 148.46 & 13 & 8.75 & 87.56 \\
\hline \multirow{2}{*}{ CD4 count } & $500-1000$ & $83(71 \%)$ & 210.36 & 36 & 43.37 & 171.13 \\
\hline & $>1000$ & $34(29 \%)$ & 130.46 & 8 & 23.52 & 61.32 \\
\hline
\end{tabular}

Table 2: Distribution of TB incidence in both prophylaxis and non-prophylaxis group.

group (26 vs 6; $\mathrm{p}=0.002$ ), which is statistically significant. Incidence of XDR-TB is more in non-prophylaxis group when compared to prophylaxis group (6 vs $1 ; \mathrm{p}=0.004)$, which is statistically significant (Table 3). Kaplan-Meier curves (Figure 2) shows a longer disease free survival for prophylaxis group than non-prophylaxis group (log-rank; $\mathrm{P}=0.002$ ).

\section{Mortality}

Among all patients, 22 from prophylaxis group and 41 from nonprophylaxis group died during the study. Most of the patients died due to increased incidence of MDR-TB and XDR-TB in the non-prophylaxis group when compared to the prophylaxis group. The crude death rate was 103 cases per 1000 person/years in prophylaxis group and 774 in the non-prophylaxis group (Table 4).

Among different age groups the mortality is more in patients of age more than 50 years when compared to any other age groups. The crude death rate is 339 cases among 1000 person/years in the age group of 40-49 years which is greater than the crude death rate in patients with age more than 50 years ( 331 person/years). The crude death rate 213 cases per 1000 person/years among males when compared to 148 cases among 1000 person/years in females.

The CD4 of range 500 to 1000 cells/ $\mu \mathrm{L}$ is having more proportion of patients when compared to CD4 of range greater than 1000 cells $/ \mu \mathrm{L}$ and the majority of the patients died belong to 500 to 1000 cells $/ \mu \mathrm{L}$ group.

Patients with low body mass effect have highest risk of deaths with tuberculosis when compared to high body mass index. KaplanMeier curves (Figure 3) indicated a longer survival for moxifloxacin completers than non-completers (log-rank; $\mathrm{P}=0.003$ ). These results were similar to the findings from Cox proportional hazards model in which moxifloxacin prophylaxis had a $60 \%$ decrease in mortality compared to non-prophylaxis (adjusted relative hazard).

\section{Discussion}

Limited data exist on safety of prophylaxis for HIV contacts to persons with MDR-TB. All MDR-TB strains are resistant to at least isoniazid and rifampin, precluding the use of these drugs for MDRTB prophylaxis in these patients. Current guidelines suggest using antibiotics to which the strain from the index case-patient is susceptible however; no randomized controlled trial has been conducted to support this recommendation. Global spread of MDR-TB necessitates identification of treatment options with acceptable safety and tolerability for persons infected with drug-resistant strains. Choosing appropriate treatment for HIV-positive persons exposed to TB is even more crucial considering the increased risk among these persons for progression from TB infection to active disease. Several factors should

\begin{tabular}{|c|c|c|c|}
\hline Tuberculosis type & $\begin{array}{c}\text { Prophylaxis } \\
\text { group }\end{array}$ & $\begin{array}{c}\text { Non-prophylaxis } \\
\text { group }\end{array}$ & N value \\
\hline $\begin{array}{c}\text { Sputum culture/smear } \\
\text { positive tuberculosis }\end{array}$ & 2 & 3 & NA \\
\hline MDR tuberculosis & 6 & 26 & 0.002 (significant) \\
\hline XDR tuberculosis & 1 & 6 & 0.004 (significant) \\
\hline Total & 9 & 35 & \\
\hline
\end{tabular}

Table 3: Tuberculosis incidence in the two groups.

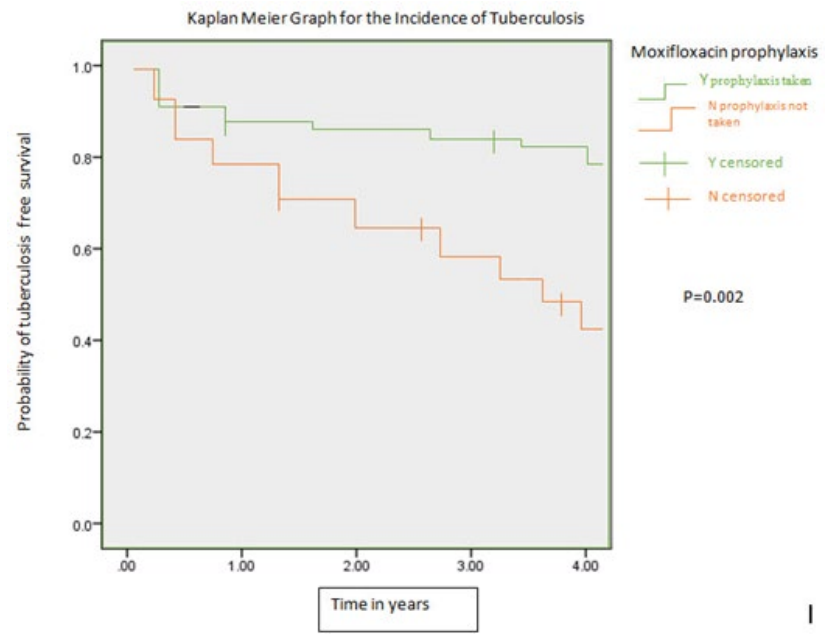

Figure 2: Kaplan Meier graph for the incidence of tuberculosis between the two groups.

\begin{tabular}{|c|c|c|c|c|c|c|}
\hline \multicolumn{2}{|c|}{ Age } & $\begin{array}{c}\text { Total } \\
\text { number }\end{array}$ & $\begin{array}{c}\text { Person } \\
\text { years }\end{array}$ & Deaths & $\begin{array}{c}\text { Incidence } \\
\text { proportion }\end{array}$ & $\begin{array}{c}\text { Death rates } \\
\text { (per 1000 } \\
\text { person/years) }\end{array}$ \\
\hline \multirow{2}{*}{ Prophylaxis } & Completed & 64 & 213.38 & 22 & 34.37 & 103.28 \\
\cline { 2 - 7 } & $\begin{array}{c}\text { Not } \\
\text { Completed }\end{array}$ & 53 & 127.44 & 41 & 77.35 & 773.58 \\
\hline \multirow{3}{*}{ Age } & $20-29$ & 33 & 118.34 & 10 & 30.3 & 84.5 \\
\cline { 2 - 7 } & $30-39$ & 26 & 97.23 & 11 & 42.3 & 113.13 \\
\cline { 2 - 7 } & $40-49$ & 34 & 67.9 & 23 & 67.64 & 338.73 \\
\cline { 2 - 7 } & $>50$ & 24 & 57.35 & 19 & 79.16 & 331.29 \\
\hline \multirow{2}{*}{ Gender } & Male & $\begin{array}{c}70 \\
(60 \%)\end{array}$ & 192.36 & 41 & 58.57 & 213.14 \\
\cline { 2 - 7 } & Female & $\begin{array}{c}47 \\
(40 \%)\end{array}$ & 148.46 & 22 & 46.8 & 148.18 \\
\hline \multirow{2}{*}{ CD4 count } & $500-1000$ & $\begin{array}{c}83 \\
(71 \%)\end{array}$ & 210.36 & 49 & 59.03 & 232.93 \\
\cline { 2 - 7 } & $>1000$ & $\begin{array}{c}34 \\
(29 \%)\end{array}$ & 130.46 & 14 & 41.17 & 107.31 \\
\hline
\end{tabular}

Table 4: Distribution of deaths both in prophylaxis group and non-prophylaxis group. 


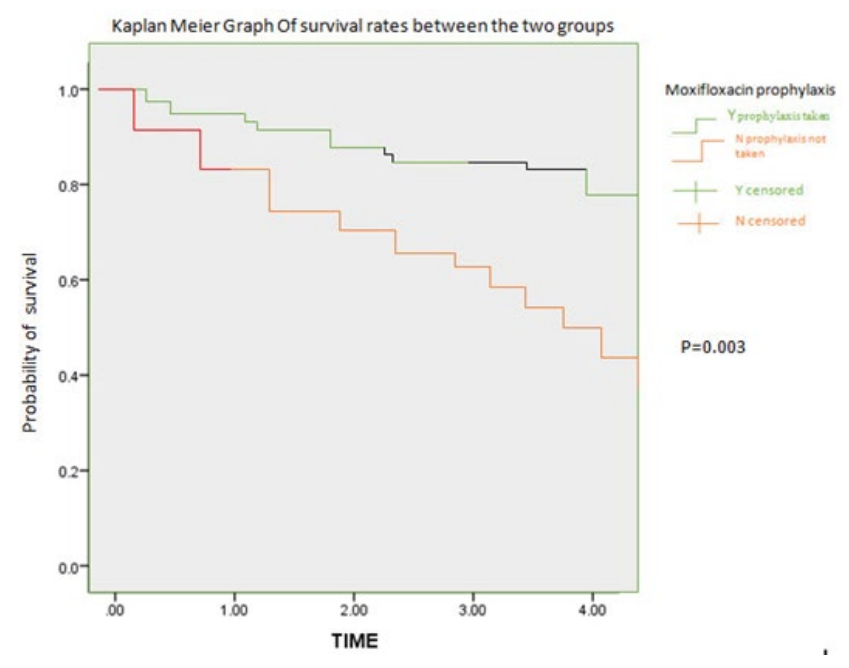

Figure 3: Kaplan Meier graph of survival rates between the two groups.

be considered in the management of HIV persons exposed to MDR-TB (i.e., contacts):

- The likelihood that the contact is newly infected with Mycobacterium tuberculosis

- The likelihood that the infecting strain of M. tuberculosis is multidrug resistant

Because, contacts of persons with infectious MDR-TB are at high risk for tuberculous infection, contacts should be rapidly identified and evaluated. Members of the immediate family, close social contacts, hospital roommates and health-care providers who shared the same indoor environment with an infectious MDR-TB patient for substantial periods are considered high-risk contacts.

Contacts who are immunosuppressed and who have a documented positive tuberculin skin test (Mantoux test with purified protein derivative - PPD) should be evaluated for preventive therapy on the basis of the susceptibility profile of the index case. Contacts who are immunosuppressed and who have no history of a positive tuberculin skin-test reaction should receive a tuberculin skin test and, if indicated, chest radiograph and sputum examination. Those who have tuberculin skin-test reactions with greater than or equal to $5 \mathrm{~mm}$ induration should be considered probably newly infected with M. tuberculosis and should be evaluated for preventive therapy, after active TB is excluded. Regardless of tuberculin skin-test results, a diagnostic evaluation should be performed if symptoms suggestive of active TB are present.

Contacts who are considered likely to be immunosuppressed who have had exposure to patients with infectious MDR-TB should be evaluated to assess the likelihood of infection with a multidrug resistant strain of $M$. tuberculosis. Several factors should be considered in this assessment:

- The infectiousness of the possible source MDR-TB case

- The closeness and intensity of the MDR-TB exposure, and

- The contact's likelihood of exposure to persons with drugsusceptible TB

This report offers suggestions for evaluating and managing persons (i.e., contacts) who have been exposed to patients with infectious MDRTB (TB due to strains of $M$. tuberculosis resistant to both isoniazid and rifampin), provides background information on alternative preventive therapy regimens with drugs other than INH or RIF, and presents considerations relevant to making a decision to offer one of these alternative regimens.

\section{Prophylaxis regimens}

Treatment of close contacts of drug resistant active cases is difficult and yet is an increasingly common clinical problem. For contacts of INH resistant index cases, INH will be ineffective [11], so 4 months of rifampicin (4RIF) is recommended [12]. Contacts of index cases with MDR-TB are recommended to receive 6 months of pyrazinamide (PZA) and a quinolone [12]. However, two observational studies have reported very high rates of toxicity and extremely poor completion due to intolerance to the drugs $[13,14]$. In both series, no patients actually completed the proposed 6 months of therapy. Based on this and with our own clinical experience, our current practice is to give moxifloxacin alone for 6 months for HIV patients with MDR-TB contact history. The rationale for this monotherapy is the published evidence that moxifloxacin can replace INH in treatment of active TB [15] during intensive phase of the treatment.

We have found that moxifloxacin in 62 patients and etambutol in 2 patients, tailored to the body weight, when given as prophylaxis, is associated with decreased incidence of TB, MDR-TB and XDR-TB. TB in HIV infected adults with contact history of known MDR-TB case. We found an association between MDR-TB prophylaxis and reduced mortality among HIV-infected persons. Before any prophylaxis therapy regimen is initiated, the diagnosis of clinically active TB must be excluded. Patients on preventive therapy should be monitored carefully for adverse reactions to the medications, evidence of active TB, and adherence to therapy.

Patients on prophylaxis therapy should be thoroughly educated about symptoms of tuberculosis and the need for immediate medical evaluation if symptoms do occur. As much as possible, alternative multidrug preventive therapy regimens should be selected, administered, and evaluated in a consistent and systematic way. All patients receiving one of these regimens should be on directly observed therapy.

\section{Safety of moxifloxacin}

Most of the patients are started on moxifloxaxin prophylaxis. Among 64 patients, 62 patients are given moxifloxacin based prophylaxis whereas 2 patients received ethambutol based on the drug culture sensitivity pattern of the index case.

The safety profile of moxifloxacin is questioned for two main reasons. First, a few initially promising fluoroquinolones have been withdrawn (e.g. gatifloxacin, temafloxacin, trovafloxaxin, sparfloxacin) and or not approved in Europe (e.g. gemifloxacin and garenoxacin) partly because of toxicity concerns, creating suspicion about the whole group. Second, the safety profile of fluoroquinolones has been challenged by the regulatory authorities, triggering

- For all approved drugs in the US, the inclusion of a 'black box warning' for tendinitis; and

- For moxifloxacin, the issue of warning about rare but serious side effects related to hepatotoxicity and severe skin reactions

In our study, all patients tolerated moxifloxacin but only one patient complains of skin rash, which subsequently subsided with treatment.

\section{Importance of the study}

This is the first trial to check the efficacy of the moxifloxacin based 
Citation: Kalyan Kumar PV, Ramakrishna G, Ramakrishna R (2016) Prospective Study of Moxifloxacin prophylaxis in Patients Suffering with HIV Having Contact History of Multidrug Resistant Tuberculosis (MDR-TB). J Pulm Respir Med 6: 377. doi: 10.4172/2161-105X.1000377

Page 6 of 6

prophylaxis for the HIV patients with contact history of MDR-TB or suspected MDR-TB patient. The basis of this trial is based on recent study of contacts treated with moxifloxacin- or levofloxacin-based prophylaxis given to the MDR-TB contacts in 2 MDR-TB out breaks [16]. The study found no serious adverse events and fewer cases of disease among those treated than those untreated.

In this study [16] of the 50 contacts, initiating moxifloxacinbased prophylaxis in the 2 outbreaks, $30(60 \%)$ completed treatment. Therapy was generally well-tolerated. Those contacts at greatest risk for development of disease may have done so during the outbreak investigation. But this study was not done on the HIV patients where the present study was done on HIV patients and shown the effectiveness of moxifloxacin prophylaxis in patients with contact history of MDR-TB.

\section{Limitation of the study}

This is a prospective observational study, so the true efficacy of the moxifloxacin prophylaxis cannot be recognized without randomized bouble blind study. All the HIV patients have CD4 count more than 500 cells $/ \mu \mathrm{L}$. But the risk of TB is more in patients having CD 4 count less than 500 cells $/ \mu \mathrm{L}$, which was not considered in this study.

\section{Conclusion}

As expanding antiretroviral therapy programs increasingly include isoniazid (INH) prophylaxis in patients with HIV. Acquired rifampicin resistance and gastrointestinal malabsorption of anti-TB drugs could, over time, contribute to further increases in the prevalence of antiTB drug resistance. Furthermore, as with any antimicrobial agent, the rapidly increasing use of isoniazid for preventive treatment in settings with a high HIV infection and TB burden could also lead to increased isoniazid resistance.

Diagnosis and treatment policies should be adapted to identify and effectively treat the increasing proportion of patients with drugresistant TB. Treatment of close contacts of drug resistant active cases is difficult and yet is an increasingly common clinical problem. For contacts of INH resistant index cases, INH will be ineffective, so an alternate therapy is required.

Prophylaxis with 6 months moxifloxacin is an effective alternative for HIV patients instead of Isoniazid prophylaxis suspected to contact with multidrug resistant tuberculosis. Six months of moxifloxacin not only decreases the incidence of tuberculosis but also decreases the mortality of these patients.

\section{References}

1. (2016) Joint United Nations Programme on HIVIAIDS: Global AIDS.

2. http://www.who.int/entity/tb/publications/global_report/en/index.html

3. WHO (2010) Multidrug and extensively drug-resistant TB (M/XDR-TB). 2010 Global Report on Surveillance and Response.

4. WHO (2016) WHO Case Definitions of HIV for Surveillance and Revised Clinical Staging and Immunological Classification of HIV-Related Disease in Adults and Children.

5. Gandhi NR, Moll A, Sturm AW, Pawinski R, Govender T, et al. (2006) Extensively drug-resistant tuberculosis as a cause of death in patients co-infected with tuberculosis and HIV in a rural area of South Africa. Lancet 368: 1575-1580.

6. Badri M, Ehrlich R, Wood R, Pulerwitz T, Maartens G (2001) Association between tuberculosis and HIV disease progression in a high tuberculosis prevalence area. Int J Tuberc Lung Dis 5: 225-232.

7. Akolo C, Adetifa I, Shepperd S, Volmink J (2010) Treatment of latent tuberculosis infection in HIV infected persons. Cochrane Database Syst Rev CD000171.

8. https://aidsinfo.nih.gov/contentfiles/lvguidelines/adult_oi.pdf

9. Bamrah S, Brostrom R, Dorina F, Setik L, Song R, et al. (2014) Treatment for LTBI in contacts of MDR-TB patients, Federated States of Micronesia, 20092012. Int J Tuberc Lung Dis 18: 912-918.

10. www.naco.gov.in/upload/2015\%20MSLNS/Annual\%20report $\% 20$ NACO_2014-15.pdf

11. Nolan CM, Aitken ML, Elarth AM, Anderson KM, Miller WT (1986) Active tuberculosis after isoniazid chemoprophylaxis of Southeast Asian refugees. Am Rev Respir Dis 133: 431-436.

12. (2000) Targeted tuberculin testing and treatment of latent tuberculosis infection Am J Respir Crit Care Med 161: S221-S247.

13. Ridzon R, Meador J, Maxwell R, Higgins K, Weismuller P, et al. (1997) Asymptomatic hepatitis in persons who received alternative preventative therapy with Pyrazinamide and Ofloxacin. Clin Infect Dis 24: 1264-1265.

14. Younossian AB, Rochat T, Ketterer JP, Wacker J, Janssens JP (2005) High hepatotoxicity of pyrazinamide and ethambutol for treatment of latent tuberculosis. Eur Respir J 26: 462-464.

15. Dorman SE, Johnson JL, Goldberg S, Muzanye G, Padayatic N, et al. (2009) Substitution of Moxifloxacin for Isoniazid during intensive phase treatment of pulmonary tuberculosis. Am J Respir Crit Care Med 180: 273-280.

16. Trieu L, Proops DC, Ahuja SD (2015) Moxifloxacin Prophylaxis against MDR TB, New York, New York, USA. Emerg Infect Dis 21: 500-503. 